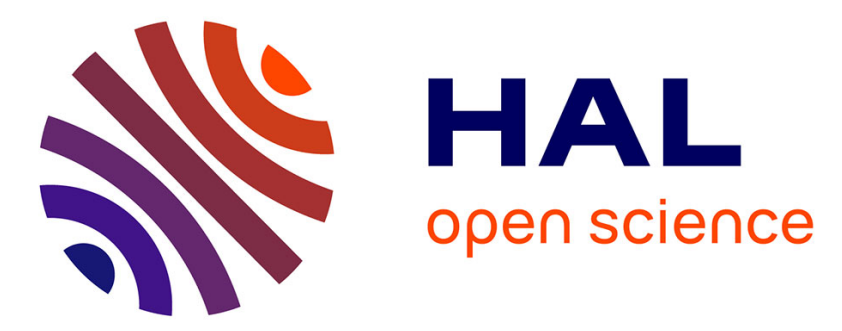

\title{
Babylift (avril 1975) : une opération militaro-humanitaire américaine pour finir la guerre du Viêtnam
}

Yves Denéchère

\section{- To cite this version:}

Yves Denéchère. Babylift (avril 1975) : une opération militaro-humanitaire américaine pour finir la guerre du Viêtnam. Guerres mondiales et conflits contemporains, 2013, 252, pp.131-143. hal03439125

\section{HAL Id: hal-03439125 \\ https://univ-angers.hal.science/hal-03439125}

Submitted on 22 Nov 2021

HAL is a multi-disciplinary open access archive for the deposit and dissemination of scientific research documents, whether they are published or not. The documents may come from teaching and research institutions in France or abroad, or from public or private research centers.
L'archive ouverte pluridisciplinaire HAL, est destinée au dépôt et à la diffusion de documents scientifiques de niveau recherche, publiés ou non, émanant des établissements d'enseignement et de recherche français ou étrangers, des laboratoires publics ou privés. 


\title{
Babylift (avril 1975) : \\ une opération militaro-humanitaire américaine pour finir la guerre du Viêtnam
}

\begin{abstract}
$\underline{\text { Résumé }}$
Pendant la guerre du Viêtnam, la mobilisation humanitaire des occidentaux s'exprime notamment par la volonté d'extirper du pays en guerre des enfants innocents. En avril 1975, alors qu'ils perdent la guerre, les Américains mènent Operation Babylift dont l'objectif est de faire sortir un grand nombre d'enfants du pays en passe de devenir communiste. Des associations françaises participent à cette évacuation marquée par le tragique accident du premier avion décollant de Saigon. Au total 120 enfants arrivent en France, près de 2000 aux États-Unis. Alors que l'épisode est peu connu en Europe, outre-Atlantique la mémoire du Babylift est entretenue par les familles adoptantes et les adoptés eux-mêmes qui présentent l'opération comme une victoire.
\end{abstract}

\section{Babylift (April 1975): \\ a U.S. military-humanitarian operation to end the Vietnam War}

\begin{abstract}
$\underline{\text { Abstract }}$
During the Vietnam War, Western humanitarian efforts were particularly concerned with extricating innocent children from the war-torn country. In April 1975, in the face of defeat, the Americans initiated Operation Babylift to bring as many children as possible out of a country at the point of falling under communist control. French associations were involved in this evacuation, marked by the tragic accident of the first aircraft taking off from Saigon. In total 120 children arrived in France, nearly 2,000 in the United States. While the episode is little known in Europe, on the other side of the Atlantic the memory of Babylift is maintained by the adoptive families and adoptees alike, who depict the operation as a victory.
\end{abstract}

\section{Introduction}

En avril 1975, alors que la République Démocratique du Viêtnam est en passe de gagner la guerre ${ }^{1}$, les États-Unis déclenchent Operation Babylift dont le but est d'évacuer le plus grand nombre possible d'enfants de Saigon. Pour les Américains, il ne saurait être question de laisser aux mains des vainqueurs communistes les métis amérasiens nés de pères soldats américains et de mères vietnamiennes. Les enfants vietnamiens en cours d'adoption par des occidentaux sont également concernés par cette évacuation qui prend la forme d'une opération militaro-humanitaire. Après des hésitations, la diplomatie française autorise des œuvres d'adoption qui travaillent au Viêtnam depuis les années 1960 à participer au Babylift, des dizaines d'enfants sont donc adoptés en France grâce à cette opération.

\footnotetext{
${ }^{1}$ Sur le déroulement de la guerre, voir : Peter Lowe (ed.), The VietnamWar, New York, St. Martin's press, 1998 ; A. J. Langguth,Our Vietnam : The War, 1954-1975, New York, Simon \& Schuster, 2000 (l'auteur a été correspondant à Saigon pour New York Times) ; Jacques Portes, Les États-Unis et la guerre du Viêtnam, Bruxelles, Complexe, 2008.
} 
Cette contribution vise à préciser les rôles des différents protagonistes occidentaux de l'opération conçue dans le but de soustraire des enfants vietnamiens aux malheurs de la guerre et de l'après-guerre communiste. En effet, le Babylift interroge autant la pratique diplomatique des États en temps de guerre que les actions des ONG au début du « second siècle de l'humanitaire $»^{2}$. Alors que l'épisode est peu connu en France, il existe aux ÉtatsUnis une histoire et une mémoire de l'opération que nous essaierons de décrypter.

Les sources mobilisées sont diverses et de natures très différentes. Les archives de Quai d'Orsay (plusieurs fonds du Centre de la Courneuve et du Centre des Archives Diplomatiques de Nantes) et des autres ministères (Archives Nationales, site de Fontainebleau) sont riches. Pour la plupart, elles ont été consultées après l'obtention de dérogations. Les archives d'associations (Terre des Hommes - France notamment), la presse écrite et audiovisuelle (INA) sont des sources complémentaires indispensables. Les ressources en ligne (archives officielles, iconographie) et les témoignages publiés sont très nombreux du côté américain mais ces dernières publications sont à aborder avec précaution. Enfin des sources orales constituées le cadre d'une étude plus large sur l'histoire de l'adoption internationale ${ }^{3}$ permettent d'entendre la parole des intermédiaires, des adoptés et des parents français.

\section{LES OCCIDENTAUX AU SECOURS DES ENFANTS DE LA GUERRE DU VIÊTNAM}

Les années 1960 voient apparaître dans les pays occidentaux des associations dont l'objectif prioritaire est de sauver des enfants victimes du sous-développement et de la guerre. Le Suisse Edmond Kaiser (1914-2000) est entièrement mobilisé pour les enfants qui souffrent, où qu'ils soient dans le monde. L'adoption lui apparaît comme un moyen efficace d'agir, une solution définitive au problème posé par l'enfance en détresse. Enfants perdus des guerres, enfants face à la famine, enfants malades et enfants handicapés concentrent toute son attention. Ainsi, à Lausanne, est créée l'association Terre des Hommes : «mouvement de combat et d'intervention immédiate et directe au secours de l'enfance meurtrie $»^{4}$. L'association Terre des Hommes - France (TDH-F) est créée en 1962. «L'accueil à vie » conçu par Terre des Hommes se définit ainsi : «l'enfant, jusqu'à sa majorité, conserve son nom, sa nationalité, sa religion d'origine. Ce n'est qu'à 21 ans qu'il lui appartiendra de choisir et d'être adopté si tel est son désir $\aleph^{5}$. En fait, et dans le cadre de la loi de 1966 réformant l'adoption, presque toutes les familles d'accueil entament très vite une procédure d'adoption plénière de l'enfant accueilli. Né dans le contexte de la guerre d'Algérie, le mouvement Terre des Hommes fait porter toute son attention sur les pays en guerre, et en premier lieu le Viêtnam.

En 1967, trois premiers enfants vietnamiens sont placés dans des familles françaises en accueil à vie. L'année suivante, ils sont déjà 41 à être extirpés du Viêtnam en guerre ; au total, en 1968, le consulat de France à Saigon délivre 92 visas pour des enfants vietnamiens ${ }^{6}$.

\footnotetext{
${ }^{2}$ Philippe. Ryfman, La question humanitaire. Histoire, problématiques, acteurs et enjeux de l'aide humanitaire internationale, Paris, Ellipses, 1999, chapitre 5.

${ }^{3}$ Yves Denéchère, Des enfants venus de loin. Histoire de l'adoption internationale en France, Paris, Armand Colin, 2011.

${ }^{4}$ Edmond. Kaiser, La marche aux enfants, Lausanne, Éditions Pierre-Marcel Favre, 1979, p.209-222. Voir également : Christophe Gallaz, Entretiens avec Edmond Kaiser, Lausanne, Favre, 1998.

${ }^{5}$ Archives de TDH-F (remerciements à Mmes André et Babinault, présidentes de l'association) ; «Historique de Terre des Hommes »; « 10 ans en France »; comptes-rendus du conseil d'administration.

${ }^{6}$ Archives du ministère des Affaires étrangères (désormais AMAE), fonds Conventions administratives et Affaires consulaires (CAAC), AC $1 n^{\circ} 6$, dépêche du consul général de France à Saigon au ministre des Affaires étrangères (MAE), 27 mars 1969.
} 
Marie-Jeanne (Minnie) Galozzi voulant faire quelque chose pour les enfants malheureux rencontre Edmond Kaiser, « un homme hors normes, passionné jusqu'au bout ». La « révolte contre la souffrance des enfants » qu'il exprime en parlant de la guerre du Viêtnam la convainc de s'engager. Elle entre donc à TDH-F et participe à différentes missions et actions dans ce pays ${ }^{7}$. Les reportages diffusés dans le cadre des actualités télévisées, les photos insoutenables d'enfants brûlés par les bombardements au napalm, les cris désespérés d'un Bernard Clavel - inspiré par Edmond Kaiser - contribuent aussi largement à mobiliser les occidentaux contre la guerre du Viêtnam ${ }^{8}$. Á Saigon, Jeanne Lê Tân, Française mariée à un Vietnamien et employée contractuelle à l'ambassade de France, prend contact avec Terre des Hommes et propose de « sortir de la misère le plus grand nombre d'enfants possible » 9 .

Face à l'augmentation du nombre de départs d'enfants, les autorités de Saigon s'émeuvent à la fin de $1968^{10}$. Un délai plus long de traitement des dossiers est imposé afin d'empêcher les convois groupés trop visibles et trop susceptibles de provoquer des réactions hostiles de la population. Une circulaire du gouvernement précise : « Envoyer des orphelins à l'étranger pour y être élevés jusqu'à leur majorité, sans se préoccuper de ce qui leur adviendra ensuite, est non seulement en contradiction avec l'esprit de nos lois, mais peut aussi porter atteinte au prestige national ». Au printemps 1970, une cinquantaine de dossiers français est en attente, le Premier ministre vietnamien tenant à viser personnellement toutes les demandes à destination de la France, «en raison de départs clandestins qui ont été décelés par les services de la sûreté vietnamienne au cours de l'année 1969 ». Des candidats français à l'adoption saisissent leurs députés qui répercutent leurs frustrations au plus haut niveau, comme par exemple William Jacson, député de Meurthe-et-Moselle, qui en 1970 écrit au ministre des Affaires étrangères pour demander l'accélération de la procédure d'adoption. Sollicité, le consul général de France à Saigon invite à la plus grande prudence, la question étant délicate car éminemment politique ${ }^{11}$. D'autant que des expatriés français, comme Jeanne LêTân, participent à ce qu'ils considèrent comme un sauvetage d'enfants mais sont vus avec suspicion par de nombreux Vietnamiens. Á chacun de ses voyages en France, elle accompagne jusqu'à neuf enfants, jamais moins de quatre ${ }^{12}$.

En 1973, Terre des Hommes - France fait venir une cinquantaine d'enfants vietnamiens $^{13}$. Au cours de l'année 1974, 600 enfants arrivent en France pour y être adoptés via Terre des Hommes et d'autres associations : le Comité de Marseille de l'Euvre de l'adoption et Le Rayon de Soleil ${ }^{14}$. Les événements militaires du début de l'année 1975, favorables aux armées nord-vietnamiennes, provoquent un véritable déferlement de demandes d'adoptions d'enfant sud-vietnamien, "parfois pour lui sauver la vie, souvent pour l'arracher aux griffes du communisme ». Le Quai d'Orsay et les œuvres sont débordés et Le Figaro s'étonne que "pour une famille française recevoir un bébé vietnamien est presque aussi difficile que d'adopter un petit Français » : vérification de l'adoptabilité, procédure juridique, attestation de la DDASS, visa de sortie du Viêtnam, visa d'entrée en France ${ }^{15}$.

\footnotetext{
${ }^{7}$ Témoignage de Mme Minnie Galozzi, militant à TDH-F puis responsable de l'association Amis des enfants du Viêtnam, recueilli par l'auteur, janvier 2010.

${ }^{8}$ Bernard Clavel, Le massacre des innocents, Paris, Robert Laffont, 1970.

${ }^{9}$ Jeanne Lê Tân, Une forêt de Bambous, Publibooks, 2008, p.39.

${ }^{10} \mathrm{AMAE} C \mathrm{CAAC}, \mathrm{AC} 1 \mathrm{n}^{\circ} 6$, dépêche du consul général de France à Saigon au MAE, 27 mars 1969, signalant que des enfants partent sans visa.

${ }^{11}$ AMAE CAAC, AC $1 \mathrm{n}^{\circ}$ 6, dépêche du consul général de France à Saigon au MAE, 14 mars 1970.

12 Jeanne Lê Tân, Op. cit., p.39-50.

${ }^{13}$ Centre des Archives Diplomatiques de Nantes (CADN), fonds Port-au-Prince, série C, n51, TDH-F, « Accueil à vie. Bilan de l'année 1973 ».

${ }^{14} \mathrm{AMAE}$ CAAC, AC $1 \mathrm{n}^{\circ} 6$, note du MAE sur l'adoption d'enfants vietnamiens par des familles françaises, s.d.

${ }^{15}$ Le Figaro, «Les longues formalités de l'adoption », 8 avril 1975.
} 
Mais l'activité la plus importante dans le domaine de l'adoption déployée par les occidentaux est le fait de grandes agences d'adoption américaines officiellement agréées par le gouvernement vietnamien : Holt International Children's Services (Holt), Traveler's AidInternational Social Services of America (TAISSA), Friends of Children of Viet Nam (FCVN), Friends For All Children (FFAC), Pearl S. Buck Foundation (PBF), World Vision Relief Organization (WVRO), Catholic Relief Services (CRS). De 1970 à 1974, elles font venir aux États-Unis 1400 enfants nés au Viêtnam ${ }^{16}$. C'est par elles que les associations françaises sont obligées de passer. Ainsi, après avoir quitté Terre des Hommes, Minnie Galozzi crée en 1970 l'association Les Amis des Enfants du Viêtnam et travaille en lien avec FCVN puis FFAC. Les deux associations américaine et française s'occupent surtout de récupérer et de placer des enfants «métis blancs » et «métis noirs » de mères vietnamiennes et de pères soldats américains ${ }^{17}$. Face à l'avancée inexorable des armées venues du NordViêtnam, en mars 1975, les ONG pressent l'administration américaine d'aider à l'évacuation des enfants vietnamiens pris en charge par leurs organisations.

\section{UNE OPÉRATION AMERICANO-AMÉRICAINE QUI COMMENCE PAR UNE CATASTROPHE}

En mars 1975, le Quai d'Orsay signale à l'ambassade à Saigon qu'après réflexion il a décidé d'accorder «des visas d'entrée en France à un certain nombre d'enfants vietnamiens adoptés par des familles françaises et qui se trouvent actuellement dans des orphelinats du centre Viêtnam, Da-Nang et Qui Nhon notamment $»^{18}$. Mais c'est trop tard, le 2 avril DaNang tombe. Alors que les États-Unis et le Canada ont obtenu des documents permettant de faire partir des enfants, les difficultés semblent plus grandes pour la France.

Le 3 avril, le Président américain Gerald Ford déclenche Operation Babylift dont l'objectif est d'évacuer les enfants métis nés de pères GI et les orphelins de guerre qui sont en cours d'adoption par des familles américaines. Deux millions de dollars sont alloués pour faire venir «le plus vite possible »aux États-Unis 2000 enfants vietnamiens. Ford donne l'ordre aux représentants américains à Saigon de tout faire pour faciliter l'opération, c'est-àdire de ne pas être trop regardant sur les formalités, et à l'armée de l'air américaine d'organiser les premiers vols immédiatement ${ }^{19}$.

Aussitôt, les œuvres d'adoption françaises qui travaillent au Viêtnam demandent à pouvoir faire partir «leurs » enfants vers les États-Unis, les autorités françaises pourraient ensuite accorder des visas d'entrée en France. Ne pouvant plus compter sur des autorités de Saigon complètement débordées, l'ambassadeur français donne le feu vert ${ }^{20}$. Dès le 4 avril, 52 enfants de l'hospice tenu par des sœurs de Saint-Paul de Chartres sont embarqués grâce à Friends for All Children dans un avion Galaxy C-5 de 1'US Air Force. Juste après avoir décollé, l'appareil connaît une avarie qui le contraint à faire demi-tour. Il s'écrase non loin de

\footnotetext{
${ }^{16}$ Agency for International Development, Operation Babylift Report (Emergency Movement of Vietnamese and Cambodian Orphans for Intercountry Adoption, April 1975), Washington, DC, juin 1975.

${ }^{17}$ Témoignage de Mme Minnie Galozzi. Friends for All Children est une émanation de Friends of Children of Viêtnam (FCVN) créée en 1974 par Rosemary Taylor, une Australienne responsible d'une œuvre d'adoption au Viêtnam.Voir Rosemary Taylor, Orphans of War: Work with the Abandoned Children of Vietnam, 1967-1975, Collins, 1988.

${ }^{18}$ AMAE Archives de la Mission pour l'Adoption Internationale (MAI), n 16, télégramme du MAE à Saigon, 27 mars 1975.

${ }^{19}$ Gerald R. Ford Presidential Library (désormais GFPL), Ann Arbor (Michigan), Fonds numérisés concernant l'Opération Babylift consultables sur http://www.fordlibrarymuseum.gov/library/exhibits/babylift/babylift.asp. Conférence de presse du 3 avril 1975 à San Diego ;President Ford's memoir : A Time to Heal: The Autobiography of Gerald R. Ford, New York, Harper \& Row, 1979, p. 252.

${ }^{20}$ AMAE MAI, n 16 , télégrammes de Saigon et du MAE, 2 et 4 avril 1975.
} 
l'aéroport de Saigon ${ }^{21}$. On dénombre plus de 150 morts,en très grande majorité des enfants, sur les 310 à 330 personnes embarquées, mais les chiffres sont très incertains...

Sous la pression des familles qui attendent l'arrivée des enfants et craignent d'apprendre qu'ils sont au nombre des victimes du Galaxy, les autorités françaises cherchent à savoir combien d'enfants ont un dossier d'adoption bouclé et sont donc susceptibles de partir du Viêtnam. Mais toute estimation est impossible, des enfants étant dispersés en province et les dossiers difficiles à saisir. Le 10 avril, le représentant français est seulement en mesure de faire un état des départs.

Départs d'enfants vietnamiens destinés à être adoptés en France (5-10 avril 1975) 22

\begin{tabular}{|c|c|l|l|l|}
\hline $\begin{array}{c}\text { Nombre } \\
\text { d'enfants } \\
\text { Destinés à } \\
\text { la France }\end{array}$ & $\begin{array}{c}\text { Date de } \\
\text { départ }\end{array}$ & Vol & Destination & \multicolumn{1}{c|}{$\begin{array}{c}\text { Organisations et aeuvres } \\
\text { concernées }\end{array}$} \\
\hline 62 & 5 avril & $\begin{array}{l}\text { Vol spécial } \\
\text { américain }\end{array}$ & San Francisco & - Friends for All Children \\
\hline 8 & 5 avril & $\begin{array}{l}\text { Vol régulier } \\
\text { Pan Am }\end{array}$ & San Francisco & $\begin{array}{l}\text { - Friends for All Children } \\
\text { - Euvre de l'adoption de Marseille }\end{array}$ \\
\hline 16 & 6 avril & $?$ & Londres & $\begin{array}{l}\text { - Aide internationale aux Immigrants } \\
\text { - Terre des Hommes }\end{array}$ \\
\hline 13 & 10 avril & $\begin{array}{l}\text { Vol régulier } \\
\text { Air France }\end{array}$ & Paris & $\begin{array}{l}\text { - Euvre de l'adoption de Marseille } \\
\text { - Rayon de Soleil } \\
\text { - Amis des Enfants du Viêtnam }\end{array}$ \\
\hline
\end{tabular}

Des enfants rescapés de l'accident du Galaxy sont convoyés par les deux premiers vols dans le cadre du Babylift. Le Président Ford et son épouse viennent les accueillir à San Francisco et encourager les personnels impliqués : pilotes, infirmières, humanitaires de Friends for All Children, etc. ${ }^{23}$ La plupart des enfants n'ont pas de visa de sortie vietnamien ni, par conséquent, de visa d'entrée en France. Leurs dossiers ont évidemment disparu dans l'accident de l'avion. Ces deux départs se sont organisés à l'insu de l'ambassade de France. Les enfants des deux convois suivants sont tous munis de laissez-passer collectifs ou individuels. Pour tous ces enfants, les familles adoptives françaises sont connues ${ }^{24}$.

Malgré la précipitation et le désordre, l'ambassadeur de France prend le temps de développer une réflexion sur l'adoption des enfants vietnamiens. Il pointe les objections qui s'expriment fortement face à l'opération Babylift que la radio de Hanoi qualifie de «trafic d'enfants ». L'accident du Galaxy renforce le sentiment de malaise des autorités de Saigon qui ne peuvent que consentir au Babylift face aux pressions américaines. "Ce douloureux problème a une dimension politique que je me dois dans les présentes circonstances de relever », conclut le diplomate ${ }^{25}$. En effet, il s'agit de ménager l'avenir et de ne pas ruiner les efforts développés par la France pour apparaître auprès de Hanoi comme un interlocuteur occidental important et utile ${ }^{26}$. La manière dont l'évacuation est organisée, avec des appareils

\footnotetext{
${ }^{21}$ Phillip R Wise, Fragile Delivery. Operation Babylift C-5A Galaxy Crash, CreateSpace Independent Publishing Platform, 2012. L'auteur est un survivant du crash; AMAE MAI, n ${ }^{\circ} 16$, correspondance du 5 avril 1975.

${ }^{22}$ AMAE MAI, $n^{\circ} 16$, correspondance des 6, 9 et 10 avril 1975

${ }^{23}$ GFPL, accueil du premier avion de Babylift à San Francisco par le Président Ford, 5 avril, 1975, photographies.

${ }^{24}$ AMAE MAI, $\mathrm{n}^{\circ} 16$, correspondance des 6, 9 et 10 avril 1975

${ }^{25}$ AMAE MAI, nº16, télégrammes de Saigon du 6 avril 1975.

${ }^{26}$ Philippe Journoud, « Diplomatie informelle et réseaux transnationaux. Une contribution française à la fin de la guerre du Viêtnam », Relations internationales, 2/2009, n 138, pp. 93-109.
} 
de l'armée américaine, d'autres affrétés par les ONG, d'autres encore des compagnies aériennes effectuant des vols réguliers sur Saigon, pose la question des relations entre l'humanitaire, le politique et militaire, les États et les $\mathrm{ONG}^{27}$. Elle n'est pas sans rappeler l'évacuation d'enfants victimes de la famine pendant la guerre du Biafra, mais celle-ci, à destination du Gabon et de la Côte d'Ivoire, était provisoire ${ }^{28}$.

L'absence de données fiables sur les embarquements est une autre caractéristique de l'opération. Il n'y a pas de listes précises, ni aucune certitude sur le nombre d'enfants embarqués. Afin de sauver leurs enfants, des familles vietnamiennes tentent par tous les moyens de les mettre elles-mêmes dans des avions. Jeanne Lê Tân qui a réussi à faire évacuer tous «ses » orphelins raconte comment Dolly, responsable d'un orphelinat et très engagée dans le Babylift, d'origine allemande et marié à un Vietnamien, rasa la tête de son fils comme cela se faisait dans certains orphelinats - pour qu'il puisse embarquer avec elle dans le Galaxy... où ils périrent tous les deux ${ }^{29}$. Une petite Vietnamienne de douze ans confie un an après son arrivée en France qu'elle est en fait la fille d'un responsable de la police de Saigon qui l'a fait partir pour la sauver en lui interdisant de révéler la vérité... ${ }^{30}$ Afin de rétablir un contrôle, le gouvernement vietnamien exige à partir du 7 avril que tous les enfants quittant le Viêtnam soient munis d'un visa de sortie, mais c'est impossible à imposer dans les faits ${ }^{31}$.

\section{ENJEUX AUTOUR DES ENFANTS DESTINÉS Á LA FRANCE}

Dès le 7 avril, une note américaine indique qu'un problème financier se posait au départ du Viêtnam des enfants destinés à la France. Friends for All Children demande aux organisations françaises la somme de 750 dollars par enfant évacué (ils seraient 120 selon la note). Alerté par les associations qui rechignent à payer, le consulat français intervient pour que cette question soit réglée plus tard, après le départ des enfants. Nous ne savons pas si elle l'a finalement été ${ }^{32}$. Mais peut-être est-ce la raison de l'arrivée à Saigon de Marguerite de Gunzbourg (née de Gramont, 1920-1998), officier de la Légion d'honneur, Croix de guerre 1939-1945 et Médaillée de la Croix-Rouge, dans le but de «faire accélérer les dossiers français ${ }^{33}$. C'est sans doute ses engagements antérieurs qui donnent à cette " grande dame» une influence certaine dans divers ministères et à l'étranger. Pour les enfants destinés à des familles françaises, le voyage de Saigon vers la côte ouest des États-Unis ne constitue que la première étape d'un long périple. Á leur arrivée, ils sont répartis dans des établissements d'accueil dépendant de l'armée américaine ou dans des hôpitaux. Certains se retrouvent dans le Colorado, d'autres à Milwaukee... Avant de les acheminer en France, il faut les regrouper, les identifier, vérifier à quelle famille ils sont destinés, établir des papiers, etc. Le tout dans les meilleurs délais ce qui n'est pas évident compte tenu des conditions.

Concernant les autorisations d'entrée en France, il y a une grande différence entre ce que le Quai d'Orsay écrit : « vous ne viserez que les seuls titulaires d'un passeport vietnamien

\footnotetext{
${ }^{27}$ Á propos du débat sur les liens entre humanitaire et militaire, voir : Bernard Kouchner, Les guerriers de la paix, Paris, Grasset, 2004 ; Rony Brauman, Humanitaire : le dilemme, Paris, Textuel, 2007 ; Michael Barnett, Empire of Humanity. A History of Humanitarianism, Cornell University Press, 2011.

${ }^{28}$ "Une trentaine de médecins, pilotes et journalistes dénoncent "le génocide au Biafra" », Le Monde, 12 février 1969 ; Michael Gould, The Struggle for Modern Nigeria. The Biafran War 1967-1970, Londres, I. B. Tauris, 2012.

${ }^{29}$ Jeanne Lê Tân, Op. cit., p.48-50.

30 Témoignage de Mme Denise Colin, responsable du secteur «Accueil à vie » de TDH-F, recueilli par l'auteur, février 2010.

${ }^{31}$ Le Figaro, «Évacuation des orphelins interrompue », 8 avril 1975.

${ }^{32}$ GFPL, notes du 7 avril 1975 destinés au conseiller spécial du Président, concernant les problèmes relatifs aux enfants destinés à des pays étrangers.

${ }^{33}$ AMAE MAI, n 16 , télégramme de San Francisco, 9 avril 1975.
} 
sur lequel a été apposé un visa français »; et ce qu'il indique par téléphone aux représentants français aux États-Unis : «il a été convenu que par souci humanitaire, nous fermions les yeux sur les visas accordés à tous [les enfants] vu l'urgence. Mais de ne pas l'écrire ${ }^{34}$... Un fonctionnaire l'a pourtant fait en griffonnant l'information sur un bout de papier, ce qui permet à l'historien de saisir une réalité qui n'était pas destinée à l'être. Paris craint que si la régularisation est trop aisée, les œuvres en profitent pour envoyer en France via les États-Unis tous les enfants des orphelinats vietnamiens. Ce qui témoigne d'une méconnaissance de Paris sur l'opération américaine.

Des responsables d'œuvres d'adoption se rendent aux États-Unis pour récupérer les enfants à placer en France. Dès le 8 avril, Mesdames Lecoanet et Galozzi des Amis des Enfants du Viêtnam sont à San Francisco. Dès l'annonce de la catastrophe du Galaxy et de la mort de son amie Dolly, Minnie Galozzi avait voulu rejoindre la Californie, mais le Quai d'Orsay le lui avait dans un premier temps interdit : les relations avec Hanoi risqueraient de pâtir d'une contribution française trop voyante à l'opération américaine. Arrivée à San Francisco, le consulat de France lui indique que désormais les autorités françaises acceptent son aide, sans qu'elle sache expliquer ce revirement ${ }^{35}$.

Á partir de listes laborieusement établies et parfois erronées, le 11 avril, treize premiers enfants partent pour Paris où les attendent des bénévoles du Rayon de Soleil, des Amis des Enfants du Viêtnam et de l'CEuvre de l'adoption de Marseille. Douze autres enfants, récupérés à Denver, partent de New York pour Paris le 16 avril, puis six le 18, cinq le 20 et douze le 21. Á San Francisco, le 17 avril, on retrouve huit enfants adoptés par des familles françaises à titre individuel, sans l'intermédiaire d'œuvres. Un enfant est opéré à cœur ouvert, d'autres doivent être hospitalisés avant de pouvoir s'envoler pour la France ${ }^{36}$.

La même confusion règne dans les aéroports parisiens. Arrivent concomitamment des enfants venant des États-Unis et d'autres qui arrivent directement de Saigon, capitale de plus en plus étouffée par les armées nord-vietnamiennes. Ainsi, le 21 avril, douze enfants en provenance de New York et dix-sept en provenance de Saigon débarquent à Roissy le même jour $^{37}$. Terre des Hommes-France, qui a refusé de participer à l'opération Babylift, accepte néanmoins de prêter son concours à l'accueil de ces enfants en jouant un rôle d'experts et de vérificateurs à la demande des autorités françaises ${ }^{38}$

Dans les derniers jours qui précédent la chute de Saigon (30 avril), de nouveaux départs ont lieu dans un contexte de panique générale. Le 28 avril arrive à San Francisco une vingtaine d'enfants destinés à des Français, beaucoup d'autres sont attendus dans les heures qui suivent ${ }^{39}$. L'emprise communiste sur tout le Viêtnam entraîne la fermeture hermétique du pays. Présente depuis plusieurs années au Viêtnam aussi bien au Nord qu'au Sud, Terre des Hommes - France fut l'une des rares organisations occidentales à pouvoir continuer à travailler pendant six mois après la chute de Saigon, et ce parce qu'elle avait refusé de participer et même de cautionner le Babylift $t^{40}$.

\section{CRITIQUES ET CONTROVERSES}

L'opération ne fait l'unanimité loin s'en faut et en premier lieu aux États-Unis. Dès le 4 avril, des professeurs d'éthique et de religion des plus grandes universités américaines

\footnotetext{
${ }^{34}$ AMAE MAI, n 16 , télégramme du MAE du 9 avril ; note téléphonique du 11 avril 1975.

${ }^{35}$ Témoignage de Mme Minnie Galozzi ; MAI, n 16 , télégramme de San Francisco du 9 avril 1975.

${ }^{36}$ AMAE MAI, n ${ }^{\circ} 16$, télégrammes de San Francisco des 16 et 21 avril et de New York du 19 avril 1975.

${ }^{37}$ AMAE MAI, $n^{\circ}$ 16, télégrammes de Saigon du 21 avril et de New York du 19 avril 1975.

${ }^{38}$ Témoignage de Mme Denise Colin.

${ }^{39}$ AMAE MAI, n¹6, télégramme de San Francisco, 29 avril 1975.

${ }^{40}$ TDH-F, conseil d'administration du 13 avril 1975.
} 
(Berkeley, Stanford...) signent une tribune dénonçant l'initiative de Ford, visant à donner bonne conscience aux Américains, et le caractère immoral de l'évacuation des enfants. D'autres critiques portent sur les difficultés d'intégration des enfants amérasiens dans un pays et une civilisation inconnus pour eux et la légitimité de les priver de leurs racines, de leur culture $^{41}$.

Les opposants à la guerre du Viêtnam, toujours plus nombreux, dénoncent le Babylift comme une ultime manifestation de l'impérialisme américain au mépris des vies humaines concernées, un «kidnapping massif ». Le 29 Avril 1975, un recours collectif est déposé par Muoi McConnell, une ancienne infirmière vietnamienne, devant la Cour fédérale de district à San Francisco, visant à interdire les procédures d'adoption jusqu'à ce que l'adoptabilité des enfants (consentement des parents biologique ou preuve de la mort des parents) soit rigoureusement établie. En effet certains enfants déclarent ne pas être orphelins et vouloir rentrer dans leurs familles. Les vérifications ont alors été renforcées ${ }^{42}$. Les choix qui ont été faits dans l'urgence sont sujets à controverse : pourquoi avoir choisi d'évacuer tel enfant plutôt que tel autre ? Pourquoi penser a priori que les Vietnamiens- fussent-ils communistes - ne prendraient pas soin de ces enfants ? D'autres ont critiqué au contraire l'insuffisance de l'opération: des enfants amérasiens n'ont pas été emmenés aux États-Unis. Et puis des Américains sont opposés à l'adoption interraciale... ${ }^{43}$ Tous ces débats ne sont pas sans rappelés ceux qui, en France comme dans les pays de la péninsule indochinoise, ont accompagné la politique de «rapatriement» des enfants eurasiens d'Indochine dans les années qui ont suivi la défaite de 1954. Déjà, les questions des critères de choix, du déracinement, du consentement des familles et des autorités locales étaient posées ${ }^{44}$.

En France, la couverture de l'événement est moindre. Une fois passée l'intensité de l'action, Le Monde avance que la trentaine de vols de l'opération Babylift a permis à plus de 2000 enfants de rejoindre les États-Unis et 200 la France, d'autres l'Australie, le Canada, quelques-uns en Suisse, Belgique, etc. ${ }^{45}$ Selon Minnie Galozzi, ce sont 120 enfants vietnamiens qui arrivent en France pendant cette période pour être adoptés. Pour Le Monde, « rarement sans doute, ne fut aussi crûment mise à nue l'ambiguïté des accès de générosité collective »; «le sort de centaines d'enfants s'est trouvé fixé en quelques heures par des bonnes volontés parfois plus soucieuses des mauvaises consciences occidentales que du sort des enfants orphelins ». Et encore, parmi les enfants, combien d'entre eux avaient encore leurs parents, de la famille ? Et comment le savoir? Dans Le Monde Diplomatique, T. D. Allman du St Anthony College à Oxford écrit: «Des avions américains qui décollent de Saigon chargés d'enfants, après y avoir débarqué leurs cargaisons de munitions. La guerre qui se perpétue, tandis que des déplacements massifs de population encouragés par la politique de Washington accumulent les complications politiques et humaines pour des années ${ }^{46}$. Afin de

\footnotetext{
${ }^{41}$ «Statement on the Immorality of Bringing South Vietnamese Orphans to the United States», 4 avril 1975, signé par six professeurs d'Université.

Consultable sur : http://pages.uoregon.edu/adoption/archive/SIBSVOUS.htm

${ }^{42}$ Maryamm George, «Operation Babylift : Mass Kidnapping ?»,Ann Arbor Sun, $1{ }^{\mathrm{er}}$ juillet 1976 ; Agency for International Development, Operation Babylift Report, op. cit.

${ }^{43}$ Charles De Benedetti (assisting author Charles Chatfield), An American Ordeal: The Antiwar Movement of the Vietnam Era, Syracuse University Press, 1990, p.379-380 ; Sherley Peck-Barnes, The War Cradle : Vietnam's children of War. Operation Babylift : The Untold Story, Vintage Pressworks, 2000.

${ }^{44}$ Yves Denéchère, "Les "rapatriements" d'enfants eurasiens en France à la fin de la guerre d'Indochine », Revue d'Histoire de l'Enfance "irrégulière », n¹4, 2012, pp.123-139.

${ }^{45}$ Pour le Canada, voir :Tarah Brookfield, Cold War comforts: Canadian women, child safety, and global insecurity, 1945-1975, Waterloo (Ontario),Wilfrid Laurier University Press, 2012, chapter 7 : The Politics of Orphans: International Adoption and Operation Babylift, p. 210-226.

${ }^{46}$ Jean-Claude Guillebaud, «L'accueil des réfugiés en France. Les enfants du Babylift », Le Monde, 23 mai 1975 ; T. D. Allman «Banqueroute morale et bonne conscience américaines », Le Monde Diplomatique, mai 1975.
} 
couper court aux polémiques et d'effacer toute traces de l'opération, le Quai d'Orsay avait demandé à Minnie Galozzi de détruire les papiers relatifs aux enfants ${ }^{47} \ldots$

\section{LES DIFFERENTES MÉMOIRES DUBABYLIFT}

Des adoptions d'enfants vietnamiens réalisées dans le but de les soustraire à la guerre, il ne reste guère de traces en France alors qu'une histoire et une mémoire de l'opération Babylift sont entretenues aux États-Unis. Plusieurs éléments peuvent être avancés pour expliquer ces positions très différentes et en premier lieu le fait évident que Babylift est une opération américaine, non française. Ensuite, dès le lendemain de la guerre, l'État français a pris soin de ne pas froisser les nouvelles autorités du Viêtnam communiste, précaution qui n'était évidemment pas de mise côté américain. Quant aux associations qui ont participé à l'opération américaine Babylift, compte tenu de la position très en retrait de l'État français et des positions critiques exprimées dans la presse, la discrétion était de mise et conseillée aux familles ayant obtenu un enfant dans ces conditions très particulières. Et puis la philosophie de l'action en direction du Tiers-monde a évolué. Terre des Hommes - France, la principale association française qui a fait adopter entre 220 et 250 enfants vietnamiens pendant la guerre, abandonne l'accueil à vie comme moyen de venir en aide à l'enfance miséreuse. Après des débats internes en 1978 l'association renonce définitivement à l'adoption internationale et recentre toute ses actions sur des formes d'aide au Tiers-monde sur place ${ }^{48}$. On comprend donc que la mémoire du Babylift n'ai pas été entretenue mais au contraire refoulée. Par ailleurs, bien peu d'auteurs français ont écrit sur le Babylift et ceux qui l'évoquent parlent de l'opération américaine, pas les enfants adoptés en France ${ }^{49}$.

Aux États-Unis le Babylift a été largement couvert par tous les médias américains, parfois comme un véritable fait de guerre ou comme un moyen de réhabiliter l'action américaine, compensant les images désastreuses de la chute de Saigon ${ }^{50}$. Ce sont d'abord les familles adoptantes concernées- en quelque sorte «bénéficiaires » de l'opération - qui ont développé et entretenu la mémoire du Babylift ${ }^{51}$. Une association les réunit régulièrement pour commémorer la geste des volontaires et des pilotes américains présentés comme des héros. Lana Noone, mère adoptive de deux enfants vietnamiens, dont une petite fille arrivée très malade et décédée un mois après son arrivée aux États-Unis, est très active pour perpétrer la mémoire du Babylift ${ }^{52}$. D'autres parents adoptifs ont écrit leurs souvenirs et justifié l'adoption humanitaire d'enfants en danger de mort ${ }^{53}$. De nombreux volontaires de l'opération ont écrit leur témoignage ${ }^{54}$. Sur les bases aériennes de la côte Ouest et dans les hôpitaux qui ont accueilli les enfants, de nombreuses plaques et stèles commémoratives ont été apposées et sont régulièrement fleuries par les familles; notamment au Continental Care Center de Denver où furent accueillis plus de 600 enfants.

\footnotetext{
47 Témoignage de Mme Minnie Galozzi.

${ }^{48}$ Yves Denéchère, « Nouvel acteur et nouveau phénomène internationaux : Terre des Hommes et l'adoption internationale (1960-1980) », in Relations Internationales, ${ }^{\circ} 142$, printemps 2010, p. 119-136.

${ }^{49}$ Olivier Todd, Cruel avril. 1975, la chute de Saigon, Paris, Robert Laffont, 1987 (réédité en 2005 sous le titre : Ce jour-là : 30 avril 1975. La chute de Saigon. Cruel avril), p.238-240.

${ }^{50}$ «Yes, there is something you can do for the Children of Vietnam», New York Times, 7 avril 1975.

${ }^{51}$ Kevin Minh Allen, « Operation Babylift : An Adoptee's Perspective », Humanist, mai-juin 2009.

${ }^{52}$ Lana Noone, Global Mom : Notes from a Pioneer Adoptive Family, Gateway Press, 2003.

${ }^{53}$ Babylift vu par des adoptantes : Pamela Chatterton Purdy, Beyond the Babylift: A Story of an Adoption, Abingdon Press, 1987 ; Allison Martin, « The Legacy of Operation Babylift », Adoption Today, Volume 2, n4, march 2000, sur : http://www.adoptvietnam.org/adoption/babylift.htm ; Andrea Warren, Escape from Saigon : How a Vietnam War Orphan Became an American Boy, Operation Babylift, Farrar, Straus and Giroux, 2004.

${ }^{54}$ Babylift vu par des volontaires : LeAnn Thieman, Carol Dey, This Must Be My Brother, Victor Books, 1995 ; Cherie Clark, After Sorrow Comes, Joy Lawrence \& Thomas Publishing House, 2000.
} 
Les $25^{\mathrm{e}}$ et $30^{\mathrm{e}}$ anniversaires de la chute de Saigon ont été propices à la mise en perspective du Babylift par les militants qui y participèrent et le considèrent comme une date importante de l'histoire de l'humanitaire. Dans les années 2000, les adoptés devenus adultes se sont penchés sur cette histoire qui est la leur. Les enfants restés au Viêtnam aussi. Kien Nguyen, né d'une mère Vietnamienne, banquière et d'un père Américain, ingénieur civil, raconte comment, en tant qu'enfant métisse, il a été considéré comme un reste indésirable du passé capitaliste après la chute de Saigon ${ }^{55}$.

En juin 2005, alors que pour la première fois le Premier ministre vietnamien était en visite aux États-Unis, des personnes adoptées, leurs familles et des volontaires du Babylift se sont rendus à Ho Chi Minh-ville ${ }^{56}$. En 2007, quelques mois après sa mort, ils ont honoré la mémoire de Gerald Ford pour sa décision pleine d'humanité de déclencher l'opération Babylift. En juillet 2013, pour le centième anniversaire de sa naissance, Lana Noone a salué son courage et sa compassion et a exprimé la gratitude de «toute la communauté du Babylift $\gg$.

Plusieurs sites Internet et blogs sont consacrés à l'adoption des enfants vietnamiens pendant la guerre et au Babylift. Des journalistes et essayistes s'y sont intéressés ${ }^{58}$, des chansons ont été écrites et des films documentaires de cinéma et de télévision ont été tournés. Operation Babylift : the Lost Children of Viêtnam réalisé en 2009 par Tammy Nguyen Lee, elle-même née à Saigon et réfugiée aux États-Unis, a obtenu de nombreuses récompenses internationales. Le film valorise les volontaires et les militants de l'opération et montre les effets de cette histoire singulière sur les personnes adoptées ${ }^{59}$. En 2008, un épisode de la série américaine Mayday qui relate les catastrophes aériennes a été consacré au crash du C5 Galaxy $^{60}$. Non loin d'Ho Chi Minh-Ville et de l'aéroport, dans la campagne vietnamienne à l'endroit où l'avion s'est écrasé, un autel dédié aux âmes errantes rappelle la mémoire des victimes.

\section{Conclusion}

Babylift a été l'une des premières opérations militaro-humanitaires impliquant des politiques, des diplomates, des militaires, des médias et des humanitaires ; des États et des ONG. Faire son histoire, c'est donc croiser différents champs de la recherche historique du temps présent et éclairer un épisode de la construction d'un espace transnational de la cause des enfants dans lequel interagissent tous les acteurs. L'opération Babylift illustre parfaitement la place importante de l'enfance sur la scène internationale de la Guerre froide, les enjeux biopolitiques qu'elle implique et le vecteur médiatique puissant qu'elle est devenue.

Interroger la mémoire que l'opération a généré de part et d'autre de l'Atlantique, c'est en prolonger l'étude jusque dans ses réalités aujourd'hui. Aux États-Unis, elle est considérée par certains comme une bataille humanitaire gagnée dans la guerre du Viêtnam perdue sur les plans militaire et politique, peut-être un des rares épisodes du conflit et de l'engagement

\footnotetext{
${ }^{55}$ Kien Nguyen, The Unwanted : A Memoir of Childhood, Back Bay Books, 2002.

${ }^{56}$ Après presque 30 ans de rupture, le premier vol commercial régulier entre les États-Unis et le Viêtnam a eu lieu le 10 décembre 2004.

${ }^{57}$ Voir http://www.vietnambabylift.org/

${ }^{58}$ Notamment :Dana Sachs, The Life We Were Given : Operation Babylift, International Adoption, and the Children of War in Vietnam, Beacon Press, 2011.

59 Tammy Nguyen Lee, Operation Babylift : the Lost Children of Vietnam, ATG Against The Grain Productions,2009, 72 min. Extraits, photographies, prix et beaucoup d'autres informations sur www.babylift.com. Le film présente notamment une vingtaine d'interviews de personnes adoptées aux ÉtatsUnis via le Babylift.

${ }^{60}$ Tim Wolochatiuk, Operation Babylift, Série Mayday n52, saison 7, 2008, 50 min.
} 
américain à valoriser. D'autres y voient la pire manière possible que les Américains avaient de finir la guerre du Viêtnam : une caricature grossière de leur impérialisme, s'imposant à des enfants innocents. Opération américano-américaine, le Babylift a néanmoins amené en France des enfants, qui ont été adoptés et sont aujourd'hui des adultes d'une quarantaine d'années qui peuvent difficilement accéder à leur histoire. Aux États-Unis, la «communauté Babylift » prépare déjà le $40^{\mathrm{e}}$ anniversaire de l'opération.

Yves Denéchère

Professeur d'histoire contemporaine Université d'Angers - UMR CERHIO

yves.denechere@univ-angers.fr 02-41-22-63-82 / 02-41-73-90-74

http://blog.univ-angers.fr/yvesdenechere/ 